\title{
Noise-induced escape flux on time-scales preceding quasistationarity
}

\author{
S.M. Soskin, V.I. Sheka, T.L. Linnik \\ V.E. Lashkaryov Institute of Semiconductor Physics, 45 pr. Nauky, 03028 Kyiv, Ukraine \\ Phone: +38 (044) 265 6175; fax: +38 (044) 265 8342; e-mail: slava@soskin.semicond.kiev.ua
}

\begin{abstract}
Noise-induced escape from the metastable part of potential is considered on time scales preceding the formation of quasiequilibrium within that part of the potential. It is shown that, counterintuitively, the escape flux may depend exponentially strongly, and in a complicated manner, on time and friction.
\end{abstract}

Keywords: noise-induced escape flux; most probable escape path.

Paper received 26.05.03; accepted for publication 17.06.03.

\section{Introduction}

The history of noise-induced escapes started in 1889 when Swedish physicist and chemist Svante Arrhenius after intensive discussion of various chemical reaction-rate data established that the rate coefficient $R$ has a form $R=A e^{-\frac{\Delta U}{T}}$, where $\Delta U$ denotes the threshold energy for activation, $\mathrm{T}$ is temperature and $A$ is a prefactor [1].

Then it was realized that escape from a metastable state can happen only via noise-assisted hopping events. Therefore the theory of escape rate was waiting for a theory of fluctuations during a long time and only in 1940 there was a seminal work of Kramers where he considered a weak noise-induced flux from a single metastable classical potential well, i.e. he considered a stochastic system [2]:

$$
\begin{aligned}
& \ddot{q}+\frac{d U}{d q}=-\Gamma \dot{q}+f_{n}(t) \\
& <f_{n}(t)>=0,<f_{n}(t) f_{n}(0)>=2 T \Gamma \delta(t), T<<\Delta U
\end{aligned}
$$

which was put initially at the bottom of a metastable potential well $U(q)$ with barrier $\Delta U$. Kramers calculated the quasistationary probability flux across the barrier, i.e. the flux established after the formation of a quasiequilibrium distribution within the well. This flux is characterized by a slow exponential decay in time $t$, an Arrhenius dependence on temperature $T$, and relatively weak dependence on friction $\Gamma$ :

$$
\begin{aligned}
& J_{q s} \equiv \alpha_{q s} e^{-\alpha_{q s} t} \\
& \alpha_{q s}=A e^{-\Delta U / T}
\end{aligned}
$$

where $A$ depends on $\Gamma$ and $T$ in a nonactivated way.

Models of type (1) are relevant to chemical reactions, SQUIDs, Josephson junctions and many other real systems, including the recently designed mechanical electrometers [3-6].

There have been many developments and generalizations of the Kramers problem but both Kramers and most of those who followed him considered only the quasistationary flux [3]. But how does the flux evolve from its zero value at the initial moment to its quasi-stationary value $J_{q s}$ ? The answer may obviously depend on initial conditions and a relevant boundary. As for the boundary, it was shown by us [7-9], that the most general qualitative features of the flux are valid for any type of bound- 


\section{S.M. Soskin et al.: Noise-induced escape flux on time-scales ...}

ary (for the sake of simplicity, we shall consider only the absorbing wall). As for the initial conditions, the most natural are those corresponding to the stable stationary state of the noise-free system, i.e. $q=q_{b}, \dot{q}=0$, where $q_{b}$ is the coordinate of the bottom of the potential well. If noise is switched on suddenly then the time evolution of the escape flux from the noise-free metastable initial state is highly relevant.

It may seem natural to assume that the flux evolution from zero to the quasistationary regime is a monotonic function without any "irregularities". Apart from the naive argument that "noise smoothes everything", this assumption appears sound because the probability distribution $\mathrm{W}$ is distinctly centered at the bottom of the well both initially and in the quasistationary stage: $W(q, \dot{q}, t=0)=\delta\left(q-q_{b}\right) \delta(\dot{q})$ while at the quasistationary stage $W$ is a narrow peak of width $\propto \sqrt{T}$ around that same state $q=q_{b}, \dot{q}=0$. Moreover, it was shown recently that, both in the underdamped and overdamped limits, the escape flux grows with time t smoothly at $t \sim t_{f}$ [10], where $t_{f}$ is time for formation of quasiequilibrium within the well:

$$
t_{f} \sim \frac{1}{\min \left(\Gamma, \omega_{o}^{2} / \Gamma\right)} \ln \frac{\Delta U}{T}
$$

Despite the above-mentioned arguments, we proved theoretically and demonstrated experimentally that there are two generic situations when the escape flux behaves in a quite complicated way [7-9]. Apart from filling the "gap" in time scales in the Kramers problem this work is motivated by growing interest in the very short time scales that is now relevant to certain experiments, such as those studying chemical reactions down to femtosecond time scales [6].

\section{Basic concept}

The previous work by Shneidman [10] on nonstationary escape rates in the Kramers problem was based on the direct solution of the Fokker-Planck equation. We apply the method of optimal fluctuation to this problem for the first time [7-9]. A transition probability density in a pathintegral representation can be written as [11]:

$$
\alpha_{t r}\left(x_{f}, t_{f} ; x_{i}, t_{i}\right)=\int_{x\left(t_{i}\right)=x_{i}} D f(t) P[f(t)] \delta\left(x\left(t_{f}\right)-x_{f}\right)
$$

where $x_{f}$ and $x_{i}$ are respectively final (at $t=t_{f}$ ) and initial (at $t=t_{i}$ ) values of dynamical variables while $P[f(t)]$ is a functional characterizing a probability density of a given noise realization $f(t)$. The dependence of $P$ on noise intensity $T$ is usually of the activation-like type $P[f(t)]=\frac{1}{Z} e^{-\frac{\tilde{S}[f]}{D_{\text {noise }}}}$, where $Z$ is a normalization factor
[11]. In particular, for a white noise [11], $\tilde{S}[f(\tau)]=\frac{1}{2} \int_{0}^{t} d \tau f^{2}(\tau)$.

Transforming from noise variables to dynamical variables $\{f \rightarrow x\}$ we derive that flux can be sought as $J(t)=A(t) e^{-\frac{S_{\min }(t)}{D_{\text {noise }}}}$, where $D_{\text {noise }} \equiv 2 \Gamma T$.

The activation energy $S_{\min }(t)$ is a minimum of the functional $\tilde{S}[x(\tau)]$ among all trajectories providing a direct transition while the prefactor $A(t)$ depends on $T$ relatively weakly. At small $T$ and short $t$, the factor $\exp \left(-S_{\min }(t) / T\right)$ depends on t much more strongly than prefactor $A$. So, we concentrate on studying $S_{\min }(t)$ which is:

$$
\begin{aligned}
& S_{\min }(t) \equiv \min [q(\tau)], \dot{q}(t)(\tilde{S}), \\
& \tilde{S}=\int_{0}^{t} d \tau L, \quad L=\left(\ddot{q}+\Gamma \dot{q}+\frac{d U}{d q}\right)^{2} /(4 \Gamma)
\end{aligned}
$$

The minimization was done over a set of escape paths $[q(\tau)]$ at a given exit velocity $\dot{q}(t)$, with a further minimization over this velocity. The path minimizing $\tilde{S}$ may be called the most probable escape path (MPEP). We describe below results obtained for the escape problem in two distinctive cases.

\section{Single-well metastable potential}

It has been proven rigorously that while $\Gamma=2 \omega_{0}\left(\omega_{0}\right.$ is the frequency of eigenoscillation in the bottom of the potential well) then $S_{\min }(t)$ and flux varies with $t$ in a stepwise manner [7-9] (Fig. 1 (b)). This conclusion is valid at any position of the absorbing wall. For weak damping $\left(\Gamma<<\omega_{0}\right)$ the characteristic scales of time and activation energy in the $n$-th step are

$t_{n} \propto \frac{n \pi}{\omega_{o}}, \quad S_{\min }\left(t_{n}\right) \propto \frac{\Delta U}{\Gamma t_{n}}, \quad n=1,2 \ldots$

Different steps correspond to different topologies of the MPEP. Thus, at short time scales $J(t)$ depends exponentially strongly both on $\Gamma$ and on $t$ (Fig. 1 (a)).

\section{The overdamped regime}

As $\Gamma$ grows, the length of a step increases while the height decreases and, at $\Gamma<<\omega_{0}$, the steps vanish. If $\Gamma$ becomes large enough we come to overdamped regime where the system may be described by twice smaller number of dynamical variables. As a consequence the activation energy can be explicitly expressed in quadratures for an arbitrary potential and arbitrary time. This allows one to find and classify all extremal paths. In this regime it is also possible to calculate the prefactor, using the numeri- 

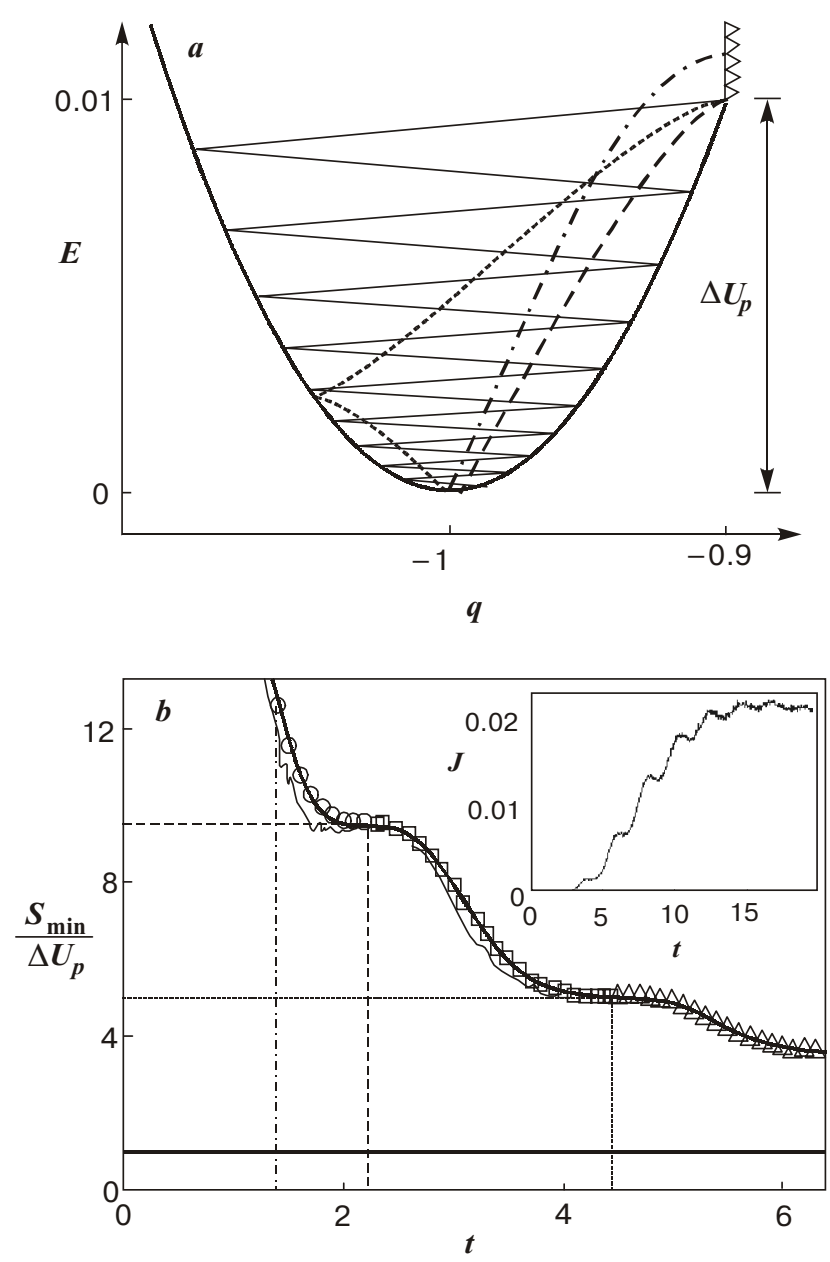

Fig. 1. $a$ - Examples of MPEPs (plotted in the energy-coordinate plane $E$ - $q$ where $E=\dot{q}^{2} / 2+U(q)$ ) for an escape from the bottom of the metastable well $U(q)=q^{2} / 2$ at $q<\sqrt{2}$ (thick solid line) to beyond the barrier at $q=\sqrt{2}(U(q)=-\infty$ at $q>\sqrt{2}$, which is equivalent to the absorbing wall indicated by triangles), at $\Gamma=0.05 ; b-$ the corresponding theoretical (thick solid line) and experimental (thin jagged line) dependences of the action $S_{\min }$ on the escape time $t$. The dashed and dotted lines indicate: in (b) the $1^{\text {st }}$ and $2^{\text {nd }}$ inflection point with $d S / d t=0$; and in $(a)$ the corresponding MPEPs. The thin solid line shows: in (b) the largetime asymptote level equal to the barrier height $\Delta U$; and in (a) the corresponding MPEP. The dash-dotted line shows in $(a)$ the MPEP corresponding to some arbitrarily chosen time $t=4.51$ and demonstrates, in particular, that the escape velocity is generally non-zero. The inset shows the experimental dependence of the flux on time, for $T=1.0$.

cal scheme suggested in [12]. Thus we provide a complete description of the noise-induced escape/transition in the overdamped system at short time scales $t<<t_{f}[13]$. The theoretical results are verified in computer simulations (Fig. 2).

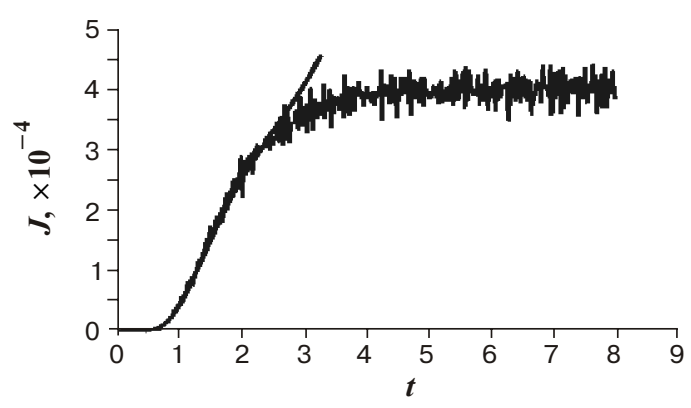

Fig. 2. The dynamics of the escape flux $J(t)$ for overdamped regime in the Duffing potential $U(q)=-q^{2} / 2+q^{4} / 4$, for $T=0.04$, measured in computer simulations (thin jagged line), and calculated by our theory (thick solid line).

\section{Multi-well metastable potential}

Unlike the single-well case, where the formation time of quasi-equilibrium is of the order of $t_{f}$, its formation in the multi-well case proceeds via two distinct stages: first, quasi-equilibrium is formed within the initial well which as in the single-well case, takes $t \sim t_{f}$ : flux $J(t)$ evolves at this stage quite similarly to the single-well case and at weak damping has steps; second, quasi-equilibrium between wells becomes established which takes exponentially longer $\left(t \sim t_{f} \exp (\Delta U / T)>>t_{f}\right)$ where $\Delta U$ means a minimal internal barrier) (Fig. 3). During the latter stage (as well as during the subsequent quasi-stationary stage), the flux $J(t)$ can be described via a solution of kinetic equations for the well populations $W_{1}$ and $W_{2}$, using the concept of constant inter-attractor transition rates $\alpha_{i j}[14,15]$ :

$$
\begin{aligned}
& J(t) \equiv W_{1} \alpha_{13}+W_{2} \alpha_{23}=\alpha_{13} e^{-t / t_{s}}+\alpha_{q s}\left(e^{-t / t_{q s}}-e^{-t / t_{s}}\right) \\
& t_{s} \approx \alpha_{12}^{-1}, \quad t_{q s} \approx \alpha_{q s}^{-1} \approx \alpha_{12} /\left(\alpha_{12} \alpha_{23}+\alpha_{21} \alpha_{13}\right) \\
& T<<U_{S 1}-U_{1}, \quad t>t_{f}
\end{aligned}
$$

The physical meaning of the two terms in (7) is easily understood. The first one corresponds to direct escapes (i.e. those ones which do not go via the bottom of well-2) and dominates before the quasi-equilibrium is established; whereas the second term, corresponding to indirect escapes, dominates in the ensuing quasi-stationary stage: it is the asymptotic part of this latter flux, $\alpha_{q s} \exp \left(-t / t_{q s}\right)$, that is called the quasi-stationary flux.

Although the coefficients $\alpha_{12}, \alpha_{21}, \alpha_{q s}$ can readily be obtained from the Kramers-Melnikov theory[2,16], $\alpha_{13}$ and $\alpha_{23}$ cannot be found in this way. One of us has developed a theory of $\alpha_{13}, \alpha_{23}$ based on the concept of optimal fluctuation $[14,15]$ :

$\alpha_{13,23}=\frac{\alpha_{q s}\left(1+\left(\Omega_{1} \Omega_{2}^{-1} \exp \left(\left(U_{1}-U_{2}\right) / T\right)\right)^{ \pm 1}\right)}{1+\left(m \exp \left(k S_{s 2 \rightarrow s 1} / T\right)\right)^{ \pm 1}}$, 


\section{S.M. Soskin et al.: Noise-induced escape flux on time-scales ...}
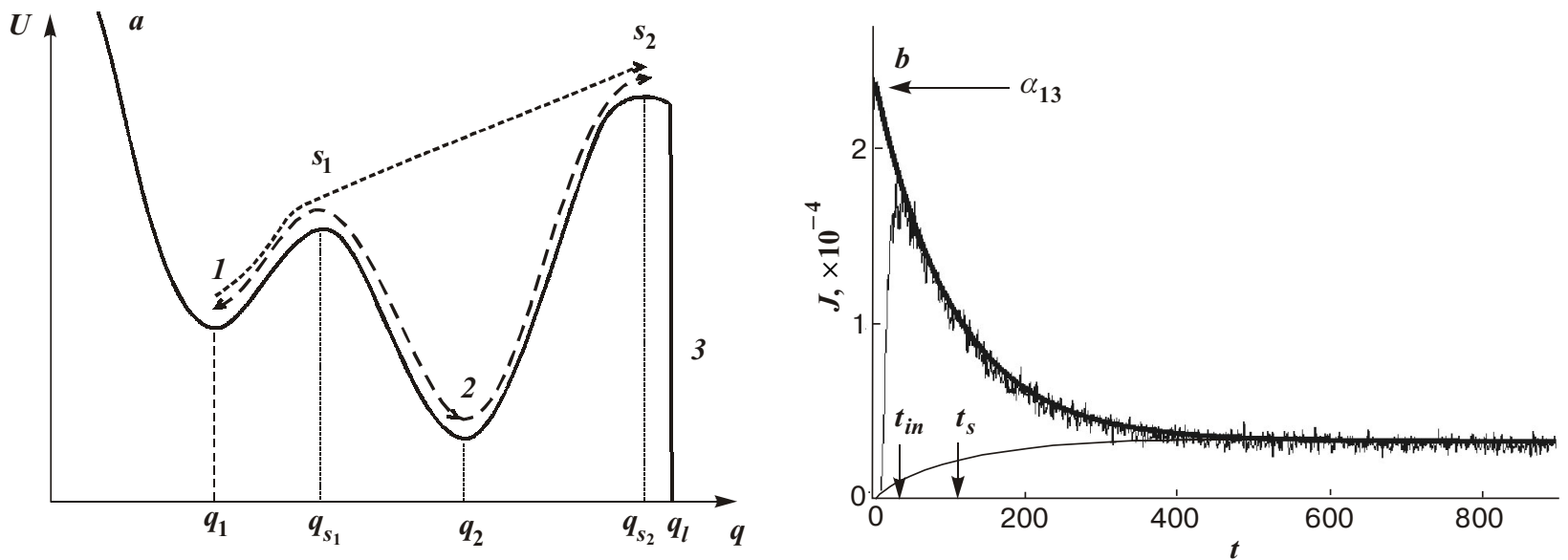

Fig. 3. $a-$ A double-well metastable potential, which describes the simplest SQUID $U(q)=0.06(q+1.5)^{2}-\cos (q), q<4.5$, $U(q)=-\infty, q>4.5$, and schematically shown direct (dotted line) and indirect (dashed line) escape path $1 \rightarrow 3$ i.e. escapes from the well 1 beyond the coordinate of absorbing wall $q=4.5 ; b$ - simulations of the dependence of the escape flux $J(t)$ on time (thin jagged line) at $\Gamma=0.15, T=0.4$ compared with the approximation of $J(t)$ by eq.(7) (thick full line); $\alpha_{12}, \alpha_{21}$ and $\alpha_{q s}$ are calculated by the Kramers-Melnikov formula [16] while $\alpha_{13}, \alpha_{23}$ are calculated by eq.(8) with $m \approx 1.1$.

where $\Omega_{1,2}$ are the frequencies of eigenoscillation in the bottom of wells 1,$2 ; k=1$ or -1 for ranges of $\Gamma$ providing the noise-free relaxation from $s_{2}$ respectively into 2 or 1 ; the action $S_{s 2 \rightarrow s 1}$ for the transition $s 2 \rightarrow s 1$ is calculated from the theory $[8,14,15]$; and $m$ is the only adjustable parameter, related to the prefactor.

As seen from eq. (8) the rates $\alpha_{13}$ and $\alpha_{23}$ depend on friction exponentially strongly, at sufficiently small temperature and have a quite complicated behavior $[8,14,15]$.

\section{References}

1. S. Arrhenius // Z.Phys. Chem., 4, p. 226 (1889).

2. H.A. Kramers, Brownian motion in a field of force and the diffusion model of chemical reactions // Physica, 7, pp. 284304 (1940)

3. P. Hanggi, P. Talkner, M. Borkovec, Reaction-rate theory: fifty years after Krasmers // Rev. Mod. Phys., 62, pp. 251-341 (1990).

4. K.K. Likharev, Dynamics of Josephson Junctions and Circuits, Gordon and Breach, Philadelphia (1986).

5. A.N. Cleland, M.L. Roukes, A nanometre-scale mechanical electrometer // Nature, 392, pp. 160-162 (1998).

6. E.W. G.Diau, J.L. Herek, Z.H. Kim, A.H. Zewail, Femtosecond activation of reactions and the concept of nonergodic molecules // Science, 279, pp.847-851 (1998).

7. S.M. Soskin, V.I. Sheka, T.L. Linnik, R. Mannella, Short time scales in the Kramers problem: a stepwise growth of escape flux // Phys. Rev. Lett., 86(9), pp. 1665-1669 (2001).
8. S.M. Soskin, V.I. Sheka, T.L. Linnik, M. Arrayas, I.Kh. Kaufman, D.G. Luchinsky, P.V.E. McClintock, R. Mannella, Noise-induced escape on time scales preceding quasistationarity: new developments in the Kramers problem // Chaos, 11(3), pp. 595-604 (2001).

9. S.M. Soskin, V.I. Sheka,T.L. Linnik, R. Mannella, Characteristic types of evolution of noise-induced escape flux at short time scales // Fluct. and Noise Lett., 1(2) , pp. L87-L95 (2001).

10. V.A. Shneidman, Transient solution of the Kramers problem in the weak noise limit // Phys. Rev., E56, pp. 5257-5264 (1997).

11. R.P. Feinman, A.R. Hibbs, Quantum mechanics and path integrals. McGraw-Hill, New York (1965).

12. J. Lehmann, P. Reimann and P. Hanggi, Surmounting oscillating barriers: path-integral approach for weak noise // Phys. Rev. E., 62(5), pp. 6282-6303 (2000).

13. S.M. Soskin, V.I. Sheka, T.L. Linnik and R. Mannella, Noiseinduced transitions in overdamped systems: short times // Noise in Complex Systems and Stochastic Dynamics, eds. L.Schimansky-Geier, D. Abbott, A. Neiman and C. Van den Broeck, Proceedings Series volume 5114, SPIE, Washington, 2003, pp. 289-300.

14. S.M. Soskin, Large fluctuations in multi-attractor systems and the generalized Kramers problem // J. of Stat. Phys., 97(3) (1999).

15. M. Arrayas, I.Kh. Kaufman, D.G. Luchinsky, P.V.E. McClintock, and S.M. Soskin, Kramers problem for a multiwell potential // Phys. Rev. Lett., 84(12), pp. 2556-2559 (2000).

16. V.I. Melnikov, the Kramers problem: fifty years of development // Phys. Rep., 209, pp. 1-71 (1991). 